12

\title{
Исследование возможности измерения радиусов зеркал приборами с датчиками волнового фронта
}

\author{
(C) А.А. Сахаров, Т.С. Пискунов, Н.В. Барышников, И.В. Животовский, Е.Е. Мухина, М.В. Вязовых \\ МГТУ имени Н.Э. Баумана, \\ 105005 Москва, Россия \\ e-mail: smx@bmstu.ru
}

Поступила в редакцию 31.05.2019 г.

В окончательной редакции 31.05.2019 г.

Принята к публикации 04.06.2019 г.

\begin{abstract}
Разработана методика измерения радиусов кривизны оптических деталей. Предложена схема прибора на основе датчика волнового фронта, реализующего минимальную погрешность измерения. Приведено теоретическое обоснование методики измерений. Рассмотрен ряд способов, позволяющих измерять радиусы кривизны оптических деталей. Проведен анализ причин увеличения погрешности измерений. Рассмотрены результаты измерения по приведенной методике макетным образцом прибора, изготовленным авторами.
\end{abstract}

Ключевые слова: радиус зеркала, метрология, датчик Шака-Гартмана, датчик волнового фронта, радиус кривизны, методика измерений, погрешности измерений, Zemax.

DOI: $10.21883 /$ OS.2019.10.48363.169-19

\section{1. Введение}

В настоящее время широкая доступность и относительная дешевизна датчиков волнового фронта (wavefront sensor, WFS) создают ситуацию, когда их использование в различных измерительных системах становится все более привлекательным [1].

В самом деле, такие системы позволяют проводить измерения различных параметров входящего излучения с очень высокой точностью, имея при этом небольшие массогабаритные характеристики и включающие контрольно-юстировочные измерительные функции по сравнению с другими [2,3] методами.

Этому способствуют в первую очередь возможности самих датчиков волнового фронта, схему работы и типовые характеристики которых приведем для оценки этого утверждения.

Итак, принцип работы WFS типа Шака-Гартмана, состоящего из матричного фотоприемника (обычно ПЗС или КМОП) и линзового растра (lens raster), описан в [4-6]. В случае регистрации плоского волнового фронта все расстояния между пятнами гартманограммы (hartmannogram) будут равны, а в случае более сложной формы эти расстояния будут различны и позволят восстановить форму волнового фронта по положению локальных нормалей.

Типовые характеристики датчика Шака-Гартмана приведены в табл. 1. Примерно таким набором параметров обладает WFS-11-26 производства компании „Активная Оптика" [7].

Из таблицы видно, что точность измерения отклонения формы волнового фронта позволяет восстановить его с очень небольшой погрешностью $[8,9]$, т.е. отличия от эталонного волнового фронта будут меньше $0.9 \mathrm{~nm}$.
Однако небольшие апертуры WFS не позволяют проводить прямые измерения параметров оптических деталей больших диаметров.

Для согласования апертур и измерения точности изготовления вогнутых и выпуклых поверхностей WFS обычно оснащается согласующей оптической системой. Типовая схема такого измерительного прибора $[10,11]$ представлена на рис. 1.

В этом приборе оптический тракт состоит из афокальной системы 4, 7 с фокусными расстояниями $f_{o b j}^{\prime}$ и $f_{\text {eye }}^{\prime}$ соответственно и фокусирующей насадки 8 с фокусным расстоянием $f_{n}^{\prime}$. WFS 8 регистрирует волновой фронт, отраженный от измеряемого зеркала 6 с радиусом $R_{\text {mir }}$, на которое приходит излучение, формируемое лазером 1 с волоконным выходом 2 и фокусируемое оптическими элементами 4 и 5 , расстояние между которыми равно $l$.

Таблица 1. Типовые характеристики датчика Шака-Гартмана

\begin{tabular}{l|c}
\hline Пространственное разрешение & $150 \mu \mathrm{m}$ \\
\hline Количество пятен гартманограммы & $4800-6400$ \\
\hline Максимальный наклон волнового фронта & $\pm 25 \mathrm{mrad}$ \\
\hline Минимальный наклон волнового фронта & $\leq 1 \mu \mathrm{rad}$ \\
\hline Максимальный радиус кривизны & $10 \mathrm{~km}$ \\
\hline Минимальный радиус кривизны & $\pm 0.34 \mathrm{~m}$ \\
\hline Повторяемость результатов $(\mathrm{RMS})$ & $0.9 \mathrm{~nm}$ \\
\hline Абсолютная точность (RMS) & $\lambda / 80$ \\
\hline $\begin{array}{l}\text { Относительная точность }(\mathrm{RMS}) \\
\text { (при угловом размере источника }<10 \mathrm{mrad})\end{array}$ & $\lambda / 500$ \\
\hline Диаметр входной апертуры & $11 \mathrm{~mm}$
\end{tabular}




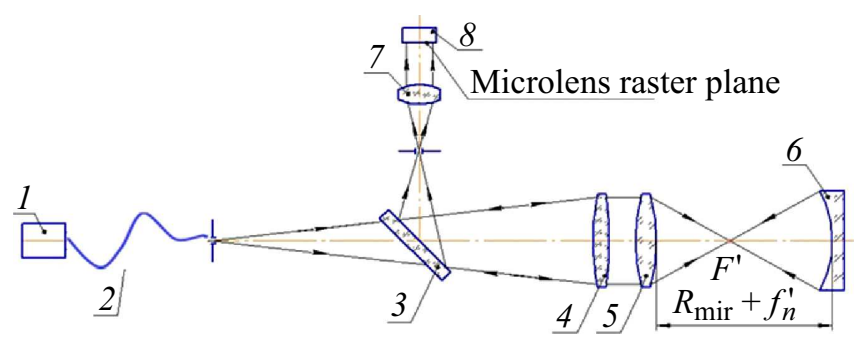

Рис. 1. Схема прибора для измерения радиуса кривизны оптической детали, начальное положение. 1 - лазер, 2 - волоконный выход, 3 - светоделитель, 4 - выходной объектив, 5 - фокусирующая насадка, 6 - измеряемая деталь, 7 объектив WFS, 8 - WFS.

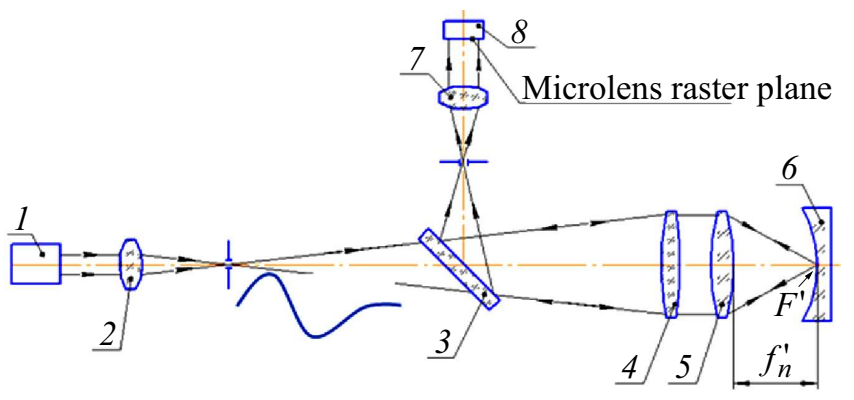

Рис. 2. Схема прибора для измерения радиуса кривизны оптической детали, конечное положение. 1 - лазер, 2 - волоконный выход, 3 - светоделитель, 4 - выходной объектив, 5 - фокусирующая насадка, 6 - измеряемая деталь, 7 объектив WFS, 8 - WFS.

Для измерения радиуса кривизны контролируемой оптической детали с помощью такого прибора существует несколько способов.

\section{2. Способы измерения радиусов зеркал}

\section{1. Способ измерения с использованием интерферометрической схемы}

Первый способ предполагает воспользоваться методикой, используемой при измерении радиусов зеркал интерферометрами Физо [12]. Суть ее состоит в том, что определяется перемещение прибора между двумя положениями. Начальное положение отображено на рис. 1 , где фокус насадки 5 совпадает с центром кривизны измеряемой детали, а конечное положение - на рис. 2 , где фокус насадки 5 совпадает с самой измеряемой поверхностью. В обоих положениях на WFS должен приходить волновой фронт с радиусом $R_{\mathrm{WFS}}=\infty$, исходя из геометрического хода лучей в указанных схемах.

В качестве тестируемых оптических деталей были выбраны пробные стекла с радиусами 37.27 и $35.65 \mathrm{~mm}$. Измерение расстояния между двумя положениями прибора (рис. 1 и 2), перемещаемого моторизованным по- движным столиком [13,14], где WFS показывает плоский волновой фронт, дает результат $34.64 \mathrm{~mm}$ для первого пробного стекла и $33.02 \mathrm{~mm}$ для второго. Погрешность $\sim 7 \%$ очень велика и не позволяет говорить о точных измерениях.

Поскольку в схеме измерения (рис. 1 и 2) в случае совпадения центра кривизны входящего в прибор волнового фронта с фокусом насадки 5 на WFS приходит плоский волновой фронт, то отличие измеренного радиуса от паспортного указывает на то, что положение прибора, показанное на рис. 1, в реальном приборе соответствует схеме, показанной на рис. 3 . То есть существует некоторое начальное рассогласование $\Delta_{\text {mis }}$ между центром кривизны измеряемого зеркала и совпадающего с фокусом насадки 2 (рис. 3 ) центра кривизны волнового фронта с радиусом $R_{\text {in }}$, входящего в прибор.

\section{2. Измерение с использованием абсолютной величины радиуса волнового фронта, измеренного WFS}

Второй способ предполагает использование непосредственно измеренного WFS радиуса кривизны волнового фронта при некотором известном рассогласовании $\Delta$ между фокусом насадки и центром кривизны измеряемой детали, при котором на WFS регистрируется предельно малый для этого датчика радиус кривизны волнового фронта. Согласно рис. 4, если предположить, что точечный источник, формируемого насадкой 1 , на-

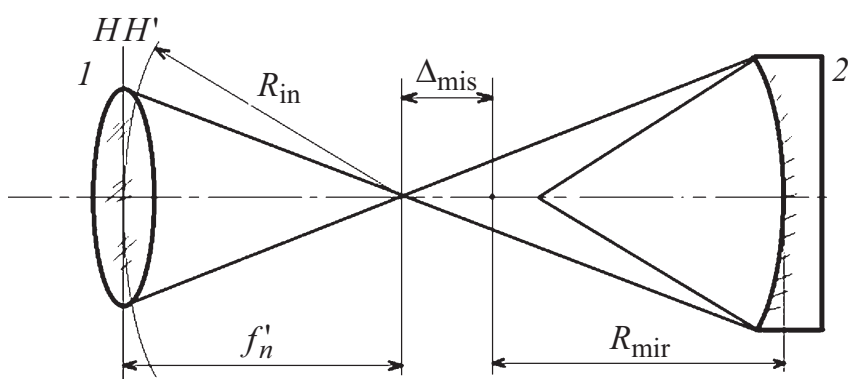

Рис. 3. Схема, описывающая начальное рассогласование. 1 - фокусирующая насадка, 2 - измеряемое зеркало.

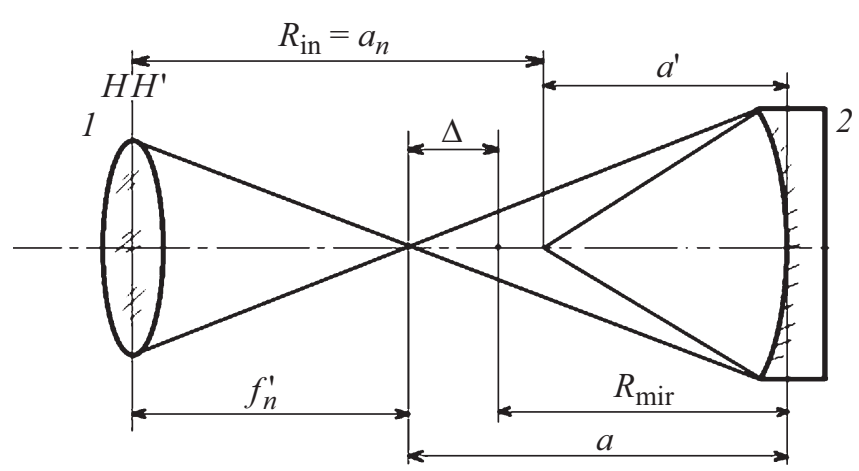

Рис. 4. Схема непосредственного измерения. 1 - фокусирующая насадка, 2 - измеряемое зеркало. 
ходится на расстоянии $a$ от измеряемой детали, то его изображение будет находиться от нее на расстоянии $a^{\prime}$. Соотношение между этими расстояниями определяется формулой (1):

$$
\frac{1}{a}+\frac{1}{a^{\prime}}=\frac{1}{f^{\prime}}
$$

учитывая, что в данном случае $f^{\prime}=R_{\text {mir }} / 2$.

В таком случае точечный источник окажется на расстоянии $R_{\text {mir }}+\Delta$ от измеряемого зеркала, а положение его относительно насадки всегда будет равно $f_{n}^{\prime}$. Просчитывая также по формуле (1) положение изображения точечного источника, создаваемого оптическими компонентами прибора (рис. 1), дальше до плоскости линзового растра WFS (где его положение будет определяться как $\left.R_{\mathrm{WFS}}\right)$, получим зависимость $R_{\mathrm{mir}}=f\left(\Delta, R_{\mathrm{WFS}}\right)$ :

$$
R_{\mathrm{mir}}=\frac{\begin{array}{c}
R_{\mathrm{WFS}}\left(f_{\text {obj }}^{\prime}\right)^{2} \Delta^{2}-f_{\text {obj }}^{\prime}\left(f_{\text {eye }}^{\prime}\right)^{2} \Delta^{2}+l\left(f_{\text {eyy }}^{\prime}\right)^{2} \Delta^{2}- \\
-f_{n}^{\prime}\left(f_{\text {eye }}^{\prime}\right)^{2} \Delta^{2}-\Delta\left(f_{\text {eye }}^{\prime}\right)^{2}\left(f_{n}^{\prime}\right)^{2}
\end{array}}{R_{\mathrm{WFS}}\left(f_{o b j}^{\prime}\right)^{2} \Delta-f_{\text {obj }}^{\prime}\left(f_{\text {eye }}^{\prime}\right)^{2} \Delta+l\left(f_{\text {eye }}^{\prime}\right)^{2} \Delta-} .
$$

Подобный метод прямой оценки фокусного расстояния линз был рассмотрен в [15], где точечный источник размещался вблизи переднего фокуса измеряемой линзы, а положение его изображения $R_{\mathrm{WFS}}$ непосредственно регистрировалось WFS.

Однако для измерения радиусов зеркал перед WFS обязательно должна стоять согласующая оптическая система (рис. 1). В этом случае вследствие рассмотренного выше начального рассогласования, обусловленного как аберрациями системы, так и негомоцентричностью источника излучения, расчет $R_{\text {mir }}$ как по формуле (2), так и по методике [15] с использованием абсолютной величины радиуса кривизны волнового фронта $R_{\mathrm{WFS}}$, измеренного датчиком, приведет к существенной погрешности при вычислении. В самом деле, формула (2) не учитывает начального рассогласования центра кривизны волнового фронта, приходящего в прибор, и геометрического центра кривизны измеряемого зеркала $\Delta_{\text {mis }}$, а также несовпадения положений фокусов и осей оптических элементов из-за погрешностей юстировки. Последние ведут, в конечном счете, также к увеличению $\Delta_{\text {mis. }}$.

В [11] было показано, что даже без учета $\Delta_{\text {mis }}$ погрешность измерения $\sigma$ при использовании абсолютного значения полученного WFS радиуса кривизны волнового фронта (формула (2)) будет составлять значительную величину. Иными словами, используя (2), погрешность измерения $\sigma$ будет определяться формулой:

$$
\sigma=\sqrt{\begin{array}{l}
\left(\frac{d R_{\text {mir }}}{d f_{\text {eye }}^{\prime}}\right)^{2}\left(\delta f_{\text {eye }}^{\prime}\right)^{2}+\left(\frac{d R_{\text {mir }}}{d f_{\text {obj }}^{\prime}}\right)^{2}\left(\delta f_{\text {obj }}^{\prime}\right)^{2} \\
+\left(\frac{d R_{\text {mir }}}{d f_{n}^{\prime}}\right)^{2}\left(\delta f_{n}^{\prime}\right)^{2}+\left(\frac{d R_{\text {mir }}}{d \Delta}\right)^{2}(\delta \Delta)^{2} \\
+\left(\frac{d R_{\text {mir }}}{d l}\right)^{2}(\delta l)^{2}+\left(\frac{d R_{\text {mir }}}{d R_{\mathrm{WFS}}}\right)^{2}\left(\delta R_{\mathrm{WFS}}\right)^{2}
\end{array}}
$$

Таблица 2. Результаты моделирования

\begin{tabular}{c|c|c|c}
\hline$R_{\text {mir }}, \mathrm{mm}$ & $\Delta_{\text {mis }}, \mathrm{mm}$ & $a, \mathrm{~mm}$ & $\Delta_{p}, \mathrm{~mm}$ \\
\hline 100 & 0.82 & 100.82 & 8.9592 \\
114.03 & 0.82 & 114.85 & 8.8621 \\
287.7 & 0.813 & 288.513 & 8.4464 \\
816 & 0.815 & 816.815 & 8.2632 \\
985.2 & 0.817 & 986.017 & 8.2442 \\
1191.2 & 0.812 & 1192.012 & 8.2351
\end{tabular}

Подставив в формулу (2) реальные параметры оптических элементов прибора $\left(f_{\text {eye }}^{\prime}=40 \mathrm{~mm}, f_{o b j}^{\prime}=200 \mathrm{~mm}\right.$, $\left.f_{n}^{\prime}=800 \mathrm{~mm}, l=30 \mathrm{~mm}\right)$, получим погрешность измерения $\sigma=50 \mathrm{~mm}$ при $R_{\mathrm{WFS}}=650 \mathrm{~mm}$ и $R_{\mathrm{mir}}=6 \mathrm{~m}$. Хотя относительная погрешность всего $0.8 \%$, СКО в $50 \mathrm{~mm}$ является значительной величиной.

Анализ (3) показывает, что при уменьшении радиуса измеряемого зеркала и при уменьшении фокуса насадки $f_{n}^{\prime}$ до $200 \mathrm{~mm}$ для сокращения общего габарита прибора погрешность вырастает до 4-7\%. Таким образом, можно утверждать, что прямые измерения, основанные на использовании формулы (2), приводят к значительным погрешностям.

\section{3. Метод калибровочной кривой}

Третий способ, описанный в [16], позволяет улучшить результаты измерений путем проведения калибровочных измерений для нескольких зеркал известного радиуса. Если на основании таких измерений строится зависимость вида

$$
\Delta=a-c e R_{\mathrm{mir}}+c \sqrt{h+e^{2} R_{\mathrm{mir}}^{2}},
$$

где $a, c, e$ и $h-$ постоянные коэффициенты, характеризующие конструктивные параметры прибора рис. 1, то погрешность измерения можно уменьшить примерно до $1 \%$ в широком диапазоне радиусов измеряемых зеркал.

\section{3. Анализ причин, приводящих к увеличению погрешности измерения}

С целью выявления причин увеличения погрешности было проведено моделирование такого метода с помощью программы Zemax [17]. В схему на рис. 1 были установлены зеркала различного радиуса и рассчитывались расстояния между задним фокусом насадки 5 и центрами кривизны этих зеркал (элемент 6 на рис. 1) при заданном радиусе волнового фронта на выходе оптической системы. Результаты такого моделирования приведены в табл. 2.

В табл. 2 представлены следующие величины: $R_{\mathrm{mir}}-$ известный радиус зеркала; $a-$ фактическое расстояние 


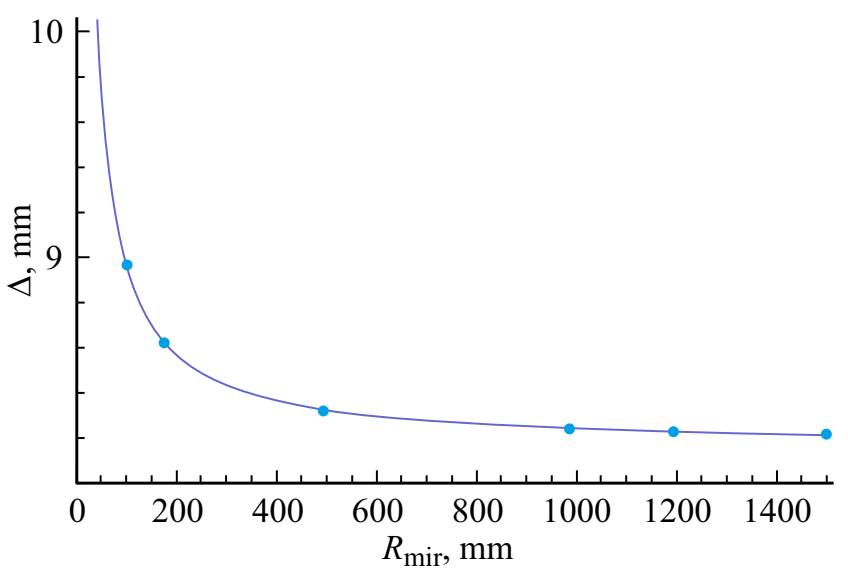

Рис. 5. Расчетная калибровочная кривая с учетом $\Delta_{\text {mis }}$, полученного в Zemax.

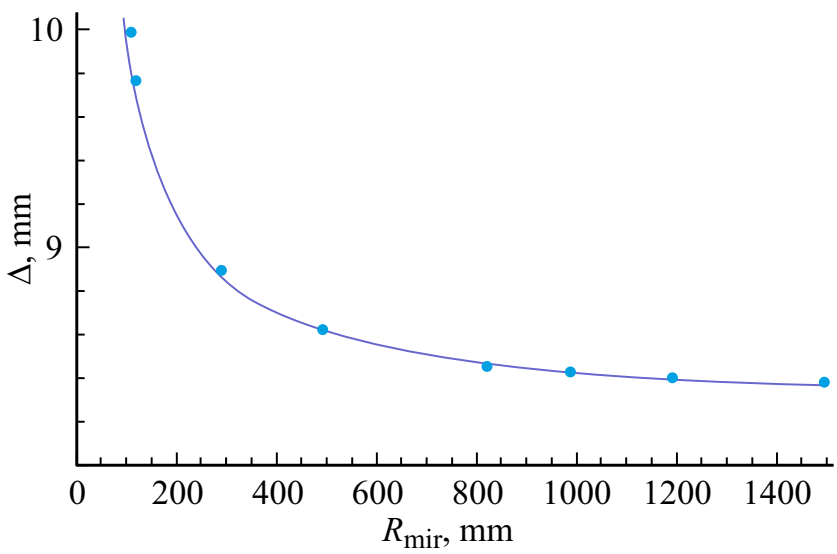

Рис. 6. Калибровочная кривая по результатам реальных измерений.

от заднего фокуса насадки до зеркала при $R_{\mathrm{WFS}}=\infty$; $\Delta_{p}$ - смещение зеркала от точки, где $R_{\mathrm{WFS}}=\infty$ до точки, где $R_{\mathrm{WFS}}=650 \mathrm{~mm} ; \Delta_{\mathrm{mis}}-$ рассогласование между центром кривизны зеркала и задним фокусом насадки при $R_{\mathrm{WFS}}=\infty$. Выявленное рассогласование невелико, однако если его не учитывать в формуле (2), то погрешность определения радиуса не позволит говорить о сколь-нибудь точных результатах.

Учесть его можно, если подставить в (2) выражение

$$
\Delta=\Delta_{p}+\Delta_{\text {mis }}
$$

Считая $R_{\mathrm{WFS}}=650 \mathrm{~mm}$ с точностью $0.02 \mathrm{~mm}$, что соответствует погрешности WFS в $1 \mathrm{~nm}$, получим результирующую табл. 3 для зеркал из табл. 2.

Если рассчитанные с учетом $\Delta_{\text {mis }}$ значения радиусов зеркал из табл. 3 подставить в методику [16], то дискретные значения смещений $\Delta$ практически точно совпадут с графиком (рис. 5) калибровочной кривой (которая показывает зависимость смещения $\Delta$ от радиуса зеркала при фиксированном радиусе кривизны волнового фронта, регистрируемого WFS), а относительная погрешность
Таблица 3. Значения радиусов кривизны зеркал

\begin{tabular}{c|c|c}
\hline $\begin{array}{c}R_{\text {mir }}, \mathrm{mm}, \\
\text { паспортный }\end{array}$ & $\begin{array}{c}R_{\text {mir }}, \mathrm{mm} \\
\text { при } \Delta=\Delta_{p}\end{array}$ & $\begin{array}{c}R_{\text {mir }}, \mathrm{mm} \\
\text { при } \Delta=\Delta+\Delta_{\text {mis }}\end{array}$ \\
\hline 100 & -39885 & 100.015 \\
114.03 & -687.1733 & 114.043 \\
287.7 & -150.7382 & 287.568 \\
816 & -111.8615 & 816.122 \\
985.2 & -108.9332 & 985.802 \\
1191.2 & -107.5833 & 1191.213
\end{tabular}

определения радиуса зеркала в этом случае составит примерно $10^{-4}$. В то же время на рис. 6 показан график смещений в зависимости от радиуса зеркала по реальным измерениям с помощью прибора, созданного в МГТУ им. Н.Э. Баумана. На нем хорошо видно, что точки реальных измерений имеют разброс относительно графика, что приводит к большим погрешностям при использовании методики калибровочной кривой.

Такое рассогласование достаточно легко выявить с помощью программ расчета оптических систем, однако на практике измерить его величину с высокой точностью в приборах с WFS, построенных по схеме рис. 1, не представляется возможным. Это обусловлено тем, что в этом случае нет какого-либо критерия совмещения геометрического центра кривизны измеряемого зеркала и заднего фокуса насадки по показаниям WFS, т.е. это совпадение не имеет особых признаков, отображаемых благодаря WFS.

\section{4. Дифференциальная методика измерений}

Более точным способом измерения радиусов кривизны оптических деталей приборами с WFS является дифференциальная методика [18]. Суть ее состоит в том, что с помощью WFS контролируется идентичность радиусов кривизны волнового фронта, приходящих на датчик для двух зеркал, одно из которых имеет известный с высокой точностью радиус. При этом смещение прибора относительно центров кривизны зеркал также измеряется с большой точностью при помощи подвижки, перемещающей прибор относительно измеряемой детали.

Такой способ измерения предполагает использование относительных характеристик WFS, а не абсолютных, что на порядок-два лучше (табл. 1). Точность в этом случае может достигать величины $0.9 \mathrm{~nm}$ rms для отклонения формы волнового фронта.

В самом деле, если точность измерения радиуса кривизны волнового фронта WFS высока настолько, что в приборе может быть зафиксировано смещение прибора относительно детали в $1 \mu \mathrm{m}$, а экспериментальные измерения подтверждают это положение, то необходимости 


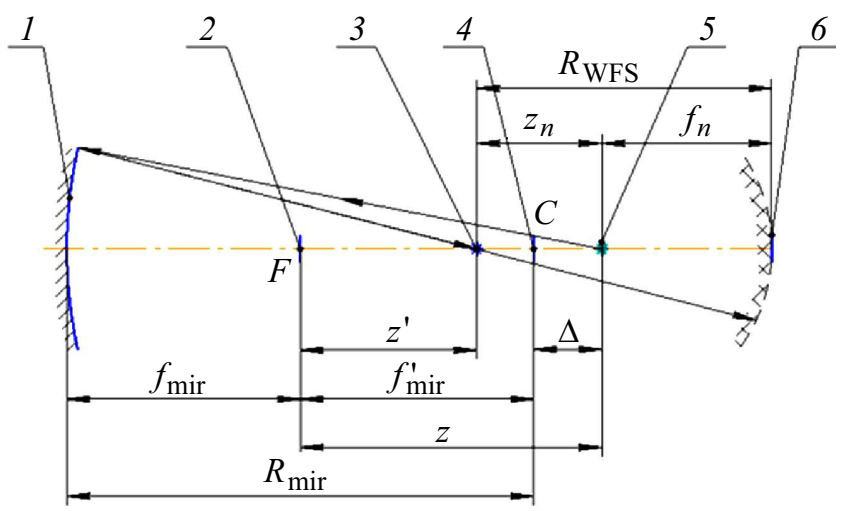

Рис. 7. Схема расчета по формуле Ньютона. 1 - измеряемое зеркало, 2 - фокус измеряемого зеркала, 3 - положение изображения точечного источника, 4 - центр кривизны измеряемого зеркала, 5 - положение точечного источника при смещении прибора, 6 - положение главных плоскостей насадки прибора.

в точном определении параметров оптических компонентов прибора, сравнивающего по дифференциальной методике два волновых фронта, нет.

Радиус кривизны волнового фронта, приходящего на прибор, можно определить, рассчитав положение изображения точечного источника относительно фокуса насадки прибора, если известно положение самого источника относительно измеряемого зеркала по формуле Ньютона:

$$
z \cdot z^{\prime}=f_{m i r}^{\prime 2}
$$

где $z$ - расстояние от точечного источника до фокуса измеряемого зеркала, $z^{\prime}$ - расстояние изображения точечного источника от того же фокуса, а $f_{\text {mir }}^{\prime}-$ фокусное расстояние этого зеркала.

Поскольку в схеме рис. 2 положение точечного источника совпадает с фокусом насадки прибора в начальном положении, то радиус кривизны волнового фронта, приходящего в прибор, будет равен фокусу насадки и $R_{\mathrm{WFS}}=\infty$. При смещении прибора из этого положения относительно зеркала на величину $\Delta\left(R_{\mathrm{WFS}} \neq \infty\right)$ можно увидеть, что

$$
z=R_{\text {mir }} / 2+\Delta
$$

а из (5) получим

$$
z^{\prime}=R_{\mathrm{mir}}^{2} / 4 z
$$

поскольку для зеркал $f_{\text {mir }}^{\prime}=R_{\text {mir }} / 2$. Величина радиуса кривизны волнового фронта, приходящего в прибор, будет равна $z_{n}+f_{n}$, что хорошо проиллюстрировано на схеме рис. 7.

Расстояние от зеркала до насадки будет равно $R_{\text {mir }}+f_{n}$ в начальном положении, а в смещенном будет либо $R_{\text {mir }}+\Delta+f_{n}$, либо $R_{\text {mir }} / 2+z^{\prime}+z_{n}+f_{n}$.

Если из последних двух выражений вычесть $f_{n}$, то их равенство все равно сохранится. Таким образом можно найти величину $z_{n}$, которая будет характеризовать пришедший в прибор радиус кривизны волнового фронта в зависимости от радиуса кривизны измеряемого зеркала:

$$
z_{n}=R_{\mathrm{mir}}+\Delta-R_{\mathrm{mir}} / 2+z^{\prime} .
$$

Подставив (6) и (7) в (8), получим

$$
z_{n}=\frac{R_{\operatorname{mir}} \Delta+\Delta^{2}}{\frac{R_{\operatorname{mir}}}{2}+\Delta} .
$$

Дифференциальная методика заключается в получении одной и той же величины $z_{n}$ для измеряемого зеркала и для эталонного благодаря контролю WFS радиуса кривизны волнового фронта, приходящего в прибор. Иными словами, если равны радиусы кривизны волновых фронтов, то равны и $z_{n}$ для сравниваемых зеркал.

Если будут известны радиус эталонного зеркала $R_{e t}$ и смещение прибора $\Delta_{e t}$, при которых измеренный датчиком радиус будет равен $R_{\mathrm{WFS}}$, то, получив такой же радиус $R_{\text {WFS }}$ и свое смещение $\Delta_{m}$ при установке измеряемого зеркала, можно найти его радиус $R_{\text {mir }}$, подставив эти значения в $(10)\left(z_{n}\right.$ для эталона и $z_{n}$ для измеряемого зеркала соответственно) и приравняв обе формулы:

$$
R_{\text {mir }}=2 \Delta_{m} \frac{2 \Delta_{e t} R_{e t}+2 \Delta_{e t}^{2}+\Delta_{m} R_{e t}-2 \Delta_{m} \Delta_{e t}}{2 \Delta_{m} R_{e t}-2 \Delta_{e t} R_{e t}-2 \Delta_{e t}^{2}+4 \Delta_{m} \Delta_{e t}} .
$$

Из табл. 2 видно, что при работе прибора по схеме рис. 1 , рассогласование между геометрическим центром кривизны зеркала и центром кривизны волнового фронта, приходящего в прибор, различается для разных радиусов зеркал. Для схемы с идеальной юстировкой, представленной в Zemax, отличия рассогласования очень малы, и поэтому такая ситуация для реального прибора теоретически может быть разрешена путем измерения смещения $\Delta$ для трех различных радиусов волнового фронта, фиксируемых датчиком: $R_{\mathrm{WFS} 1}, R_{\mathrm{WFS} 2}, R_{\mathrm{WFS} 3}$. В этом случае получим систему из трех независимых уравнений с тремя неизвестными, аналогичными уравнению $(10)$, из которой можно будет найти $R_{\text {mir }}$, начальное рассогласование для эталона и измеряемого зеркала.

В ходе экспериментальных измерений была выявлена зависимость указанного рассогласования не только от радиуса кривизны измеряемого зеркала, но и от смещения $\Delta$. Поскольку в реальном приборе рассогласование для начального положения различных зеркал оказывается очень большим - 4.8-5.2 mm, считать его постоянной величиной для различных смещений $\Delta$ невозможно, так как это приводит к существенному увеличению погрешности измерения.

Решить описанную проблему можно, попытавшись уменьшить рассогласование путем сложных схем юстировки и использованием дорогостоящего калибровочного оборудования так, чтобы хотя бы отличия этой величины для разных зеркал были бы небольшим, и его можно было бы считать постоянным. Однако рассогласование, вызванное аберрациями оптических компонентов, останется в любом случае. 


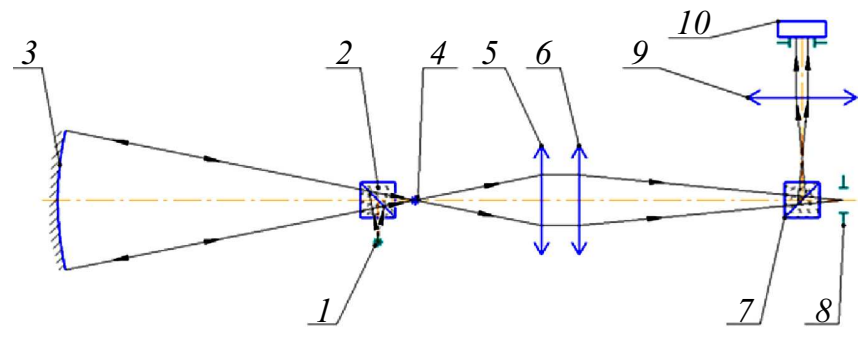

Рис. 8. Схема прибора с отсутствием начального рассогласования. 1 - положение точечного источника, 2 - светоделитель, 3 - измеряемое зеркало, 4 - центр кривизны измеряемого зеркала, 5 - фокусирующая насадка, 6 объектив 1,7 - светоделитель, 8 - юстировочная диафрагма, 9 - объектив $2,10-$ WFS.
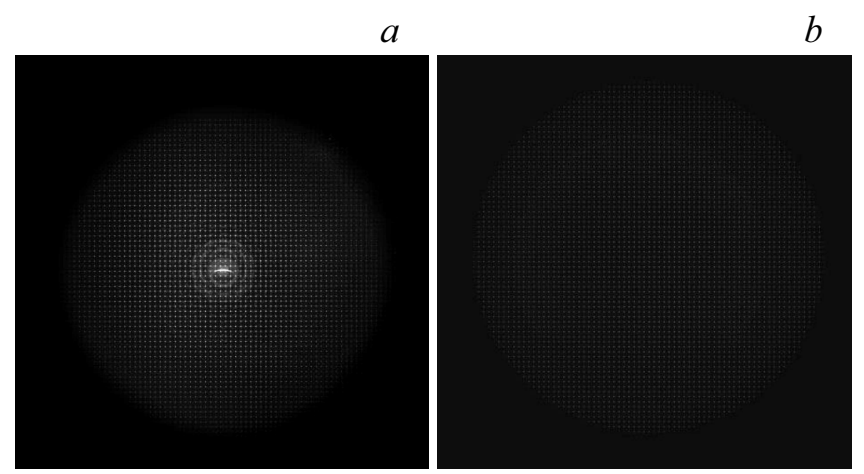

Рис. 9. Гартманограммы начального положения. $a-$ прибор по схеме рис. $1, b-$ прибор по схеме рис. 8.

\section{5. Схема прибора без рассогласования}

Кардинальное же решение состоит в том, чтобы вообще устранить такого рода рассогласование. Это можно сделать, изменив схему прибора так, чтобы в центр кривизны измеряемого зеркала помещался непосредственно сам точечный источник, а не его проекция через оптическую систему прибора. Тогда схема прибора будет выглядеть так, как представлено на рис. 8.

Точечный источник 1 вынесен за пределы проекционной части оптической системы прибора, состоящей из фокусирующей насадки 5 и первого объектива 6 афокальной системы (6 и 9), и помещается непосредственно в центр кривизны измеряемого зеркала 3 с помощью светоделительного элемента 2. Последний позволяет совместить центр кривизны зеркала с фокусом насадки 5 в точке 4. В итоге на WFS формируется плоский волновой фронт, который указывает на точное совпадение центра кривизны волнового фронта, приходящего в прибор (в фокусирующую насадку), центра кривизны измеряемого зеркала и точечного источника. Для удобства юстировки прибор имеет светоделительный кубик 7 и точечную диафрагму 8 .
В такой схеме принципиально отсутствует рассогласование $\Delta_{m i s}$, обусловленное аберрациями оптической системы, однако небольшая неточность совмещения точечного источника и центра кривизны измеряемого зеркала $\delta$ вследствие погрешностей юстировки остается. В отличие от первой схемы прибора эта неточность будет постоянной величиной для каждого измеряемого зеркала.

Такая схема позволила одновременно избавиться от паразитной засветки центральной области гартманограммы ввиду отсутствия блика, отраженного от поверхности насадки 5, обращенной к объективу 4 (рис. 1). Это обусловлено тем, что при выборе большого фокусного расстояния насадки, о котором будет идти речь ниже, поверхность оптического элемента имеет большой радиус кривизны и отраженный от нее плоский волновой фронт, пришедший после объектива 4 , будет иметь маленькую расходимость, а попадая в апертуру WFS, создает паразитную засветку. Гартманограммы для рассмотренных схем построения прибора приведены на рис. 9.

Поскольку при дифференциальной методике в расчетах не используются параметры оптической системы прибора и непосредственно характеристики WFS, основной погрешностью, влияющей на точность измерения радиусов зеркал в такой схеме, будет относительная погрешность измерения перемещения прибора относительно измеряемой детали $\delta \Delta / \Delta$, где $\delta \Delta-$ погрешность определения перемещения. Для ее уменьшения требуется либо повысить разрешение устройства перемещения, либо увеличить расстояние, на которое перемещается прибор относительно измеряемой детали. Повысить разрешение устройства можно только тогда, когда WFS позволит отследить перемещение прибора на это минимально разрешаемое расстояние. Экспериментами подтверждено, что при погрешности измерения отклонения волнового фронта в $1 \mathrm{~nm}$ минимально отслеживаемый шаг будет составлять $1 \mu \mathrm{m}$, если использовать реальные параметры оптических элементов прибора, изготовленного по схеме рис. $8\left(f_{9}^{\prime}=40 \mathrm{~mm}, f_{6}^{\prime}=200 \mathrm{~mm}\right.$, $f_{5}^{\prime}=500 \mathrm{~mm}$, а расстояние между 5-м и 6-м компонентами $l=30 \mathrm{~mm}$ ). Найти WFS, позволяющий отследить перемещение с меньшей погрешностью сложно, так как его погрешность в $1 \mathrm{~nm}$ близка к пределу современных датчиков. Устройство перемещения с разрешением менее $1 \mu \mathrm{m}$ также найти весьма затруднительно, поскольку либо диапазон перемещений будет небольшой, либо его стоимость будет очень высока.

Увеличение предельного перемещения при проведении измерений ограничено в первую очередь динамическим диапазоном WFS (фактически определяется минимально регистрируемым датчиком радиусом кривизны волнового фронта), а также соотношением фокусов афокальной системы $f_{9}^{\prime}$ и $f_{6}^{\prime}$ величиной фокусного расстояния насадки $f_{5}^{\prime}$. Выбрав величину последнего $500 \mathrm{~mm}$, получаем диапазон перемещений $\sim 18 \mathrm{~mm}$ для 
Таблица 4. Зависимость погрешности измерения от неточности установки

\begin{tabular}{c|c|c|c|c|c}
\hline$\delta, \mu \mathrm{m}$ & 10 & 30 & 50 & 100 & 150 \\
\hline$\sigma, \mathrm{mm}$ & 8.775 & 26.326 & 43.876 & 87.752 & 131.629
\end{tabular}

Таблица 5. Результаты измерения радиусов кривизны зеркал

\begin{tabular}{c|c|c|c|c}
\hline$R_{\text {mir }}, \mathrm{mm}$ & 174.96 & 493.3 & 985.2 & 1191.2 \\
\hline$\sigma, \mathrm{mm}$ & 0.125 & 0.958 & 3.656 & 5.395
\end{tabular}

100 mm-зеркала и относительную погрешность измерения перемещения $\delta \Delta / \Delta=5.5 \cdot 10^{-5}$. При увеличении $f_{5}^{\prime}$ диапазон перемещений растет, но увеличиваются габариты прибора и сильно уменьшается область засветки измеряемой детали (уменьшение апертурного угла). По этой причине выбор основных параметров оптической системы является своего рода компромиссом между различными выходными характеристиками готового прибора.

Попробуем теперь оценить погрешность измерения радиуса кривизны тестируемого зеркала. Ее можно рассчитать, воспользовавшись следующим известным выражением:

$$
\delta=\sqrt{\left(\frac{d R_{\mathrm{mir}}}{d \Delta_{e t}} \delta \Delta\right)^{2}+\left(\frac{d R_{\mathrm{mir}}}{d \Delta_{m}} \delta \Delta\right)^{2}+\left(\frac{d R_{\mathrm{mir}}}{d R_{e t}} \delta R_{e t}\right)^{2}},
$$

где $\delta \Delta-$ погрешность измерения перемещения столика прибора, а $\delta R_{e t}-$ погрешность измерения радиуса эталонного зеркала.

Однако при измерениях необходимо учитывать, как было сказано выше, тот факт, что начальное положение как эталонной, так и измеряемой детали установлено с некоторой погрешностью $\delta$ относительно точечного источника, которая изначально не известна. При включении этого первоначального смещения в расчет получим следующую величину перемещения прибора $\Delta$, равную соответственно $\Delta_{e t}+\delta_{e t}$ для эталона и $\Delta_{m}+\delta_{m}$ для измеряемой детали.

В этом случае погрешность определения перемещения будет включать в себя эту начальную неточность установки $\delta$ как для эталона, так и измеряемой детали, и тогда формулу (11) можно записать в следующем виде:

$$
\delta=\sqrt{\begin{array}{l}
\left(\frac{d R_{\mathrm{mir}}}{d \Delta_{e t}} \delta\left[\Delta+\delta_{e t}\right]\right)^{2}+ \\
+\left(\frac{d R_{\mathrm{mir}}}{d \Delta_{m}} \delta\left[\Delta+\delta_{m}\right]\right)^{2}+\left(\frac{d R_{\mathrm{mir}}}{d R_{e t}} \delta R_{e t}\right)^{2}
\end{array}}
$$

Анализ погрешности измерения из выражения (12) приводит к следующим результатам, отображенным в табл. 4.
Хорошо видно, что при $\delta>10 \mu \mathrm{m}$ погрешность становится неприемлемо большой, что говорит уже о высокой степени неопределенности измерений. Однако погрешность $\delta$ является систематической и может быть вычислена. Подставив $\delta$ для эталона и измеряемого зеркала в формулу (10), получим уравнение с тремя неизвестными $\left(R_{\mathrm{mir}}, \delta_{m}\right.$ и $\left.\delta_{e t}\right)$, т. е., как и говорилось, по дифференциальной методике (разд. 4) для нахождения нужных неизвестных достаточно будет как минимум 3 измерения.

\section{6. Усреднение результатов измерений}

Стоит отметить, что измерение $\Delta_{e t}$ и $\Delta_{m}$ происходит в различные моменты времени, когда условия измерений (температура, освещенность, флуктуации параметров лазерного источника) могут различаться, поэтому точное решение системы из трех уравнений без учета этих факторов приводит к погрешности в несколько миллиметров. Эту погрешность можно существенным образом снизить, сделав серию измерений для различных $R_{\text {WFS }}$ и решив полученную систему уравнений, например, методом наименьших квадратов. В результате такой методики уменьшается влияние указанных выше случайных факторов на погрешность измерения.

Суть ее состоит в том, что, получив зависимость $z_{n}=f\left(\Delta_{e t}, R_{e t}\right)$ для эталонного зеркала, можно согласно формуле Ньютона найти такую же зависимость $z_{n}=f\left(\Delta_{m}, R_{\text {mir }}\right)$ для измеряемого зеркала, которая полностью совпадет с эталонной при подборе $R_{\text {mir }}$.

Примером такого подхода может служить описанный в [15] процесс измерения фокусного расстояния линз путем многократного изменения радиусов кривизны волновых фронтов, приходящих на WFS. Однако напрямую воспользоваться таким методом невозможно, поскольку, как сказано вначале статьи, при использовании реальной оптической системы нет достоверной математической связи между радиусом кривизны волнового фронта на входе прибора и $R_{\mathrm{WFS}}$, регистрируемым WFS. Поэтому и выбрана дифференциальная методика, где используется эталонное зеркало с точно измеренным радиусом.

В качестве функции минимизации ошибки также нельзя использовать выражение, указанное в [15], поскольку формула расчета радиуса (фокуса) оптической детали отличается от таковой в [15].

С учетом этого самым простым вариантом может быть выражение, позволяющее найти минимальную разницу между выражениями (9) для эталона и измеряемого зеркала соответственно:

$$
z_{n}=\frac{2 f_{e t} \Delta_{e t}+\Delta_{e t}^{2}}{f_{e t}+\Delta_{e t}}
$$

по всей серии измерений, перебором с небольшим шагом величин $R_{\mathrm{mir}}, \delta_{m}$ и $\delta_{e t}$, путем суммирования по абсолютной величине разности квадратов формул (9) для эталона и измеряемого зеркала. Это необходимо для 
Таблица 6. Относительная погрешность измерения радиусов кривизины зеркал

\begin{tabular}{c|c|c|c|c}
\hline $\begin{array}{c}\text { Паспортный радиус } \\
\text { кривизны } \\
\text { зеркала } R_{\text {mir }}, \mathrm{mm}\end{array}$ & $\begin{array}{c}\text { Измеренный радиус } \\
\text { кривизны зеркала } \\
R_{\text {mir, mm схема рис. } 8}\end{array}$ & $\begin{array}{c}\text { Относительная погрешность } \\
\text { измерения радиуса } \\
\text { зеркала по схеме рис. 8 }\end{array}$ & $\begin{array}{c}\text { Измеренный радиус } \\
\text { кривизны зеркала } \\
R_{\text {mir, mm схема рис. 1 }}\end{array}$ & $\begin{array}{c}\text { Относительная погрешность } \\
\text { измерения радиуса } \\
\text { зеркала по схеме рис. 1 }\end{array}$ \\
\hline 287.7 & 287.65 & 0.00017 & 287.11 & 0.002 \\
493.13 & 493.3 & 0.00034 & 492.31 & 0.0018 \\
985.2 & 985.12 & 0.00008 & 987.47 & 0.0023 \\
1191.2 & 1191.15 & 0.000042 & & - \\
1493.3 & 1493.05 & 0.00017 & & -
\end{tabular}

независимости от знака разности указанных выражений. Следовательно, задав в цикле перебора значения неизвестных величин, необходимо будет искать минимум следующего выражения:

$$
\begin{aligned}
\Sigma_{i=1}^{N}= & \mid\left[\frac{2 f_{e t}\left(\Delta_{e t}+\delta_{e t}\right)+\left(\Delta_{e t}+\delta_{e t}\right)^{2}}{f_{e t}+\left(\Delta_{e t}+\delta_{e t}\right)}\right]^{2} \\
& -\left[\frac{2 f_{m}\left(\Delta_{m}+\delta_{m}\right)+\left(\Delta_{m}+\delta_{m}\right)^{2}}{f_{m}+\left(\Delta_{m}+\delta_{m}\right)}\right] \mid,
\end{aligned}
$$

где $N$ - количество измерений в серии.

Таким образом, все искомые неизвестные величины находятся как параметры неявно заданной функции, а саму функцию находят путем нахождения наименьшего отличия от известной ,родительской“ функции (такой же зависимости, как и искомая, но с известными параметрами). Это означает, что чем больше будет получено разных значений для ,родительской“ и искомой функции, тем значительнее можно уменьшить погрешность измерения $R_{\text {mir. }}$ Подробно подобная методика описана в [19]. В этом случае погрешность измерения при выборке из $N$ точек будет определяться по формуле:

$$
\begin{aligned}
\delta_{N} & =\frac{1}{\sqrt{N-m}} \\
& \times \sqrt{\left(\frac{d R_{\mathrm{mir}}}{d \Delta_{e t}} \delta \Delta\right)^{2}+\left(\frac{d R_{\mathrm{mir}}}{d \Delta_{m}} \delta \Delta\right)^{2}+\left(\frac{d R_{\mathrm{mir}}}{d R_{e t}} \delta R_{e t}\right)^{2}},
\end{aligned}
$$

где $m$ - число степеней свободы в функции минимизации ошибки, характеризующей наилучшее совпадение (отличие измеренной зависимости от „родительской“) путем подбора нескольких параметров. Поскольку искомыми являются 3 параметра, то число степеней свободы для выбранной методики $m=3$ [19].

Стоит отметить, что частные производные в каждой точке измерений не равны, поэтому есть смысл говорить о среднем значении этих производных на всем интервале измерения.

Также необходимо добавить, что формула (13) позволяет учесть измерения вблизи нулевых значений, когда из-за различных флуктуаций параметров окружающей среды могут получиться разные по знаку значения $z_{n}$ для одного и того же $R_{\mathrm{WFS}}$. Она достаточно чувствительна к отдельным измерениям, но при ограничении интервала поиска $R_{\text {mir }}$ результат либо точно попадает в значение паспортного радиуса (для исследованных зеркал), причем при относительно небольшом количестве измерений, либо выходит за пределы интервала (в случае несовпадения условий измерения эталона и исследуемого зеркала). Экспериментальные исследования достаточно хорошо подтверждают эту ситуацию.

\section{7. Результаты измерений}

В рамках описанного исследования, следуя описанной в разд. 6 методике, в МГТУ им. Н.Э. Баумана были проведены измерения с помощью прибора, схема которого представлена на рис. 8, для нескольких зеркал с различными радиусами кривизны. Параметры оптических элементов, соответствующие схеме рис. 8, были следующие: $f_{5}=500 \mathrm{~mm}, f_{6}=200 \mathrm{~mm}, f_{9}=40 \mathrm{~mm}$. WFS имел динамический диапазон по параметру „дефокусировка“ $15-20 \mu \mathrm{m}$, а повторяемость на уровне $0.9 \mathrm{~nm}$ [7].

Результаты такого исследования для нескольких зеркал с учетом погрешности перемещения прибора $\delta \Delta=1 \mu \mathrm{m}$ (паспорт) и погрешности измерения радиуса эталонного зеркала $\delta R_{e t}=1 \mu \mathrm{m}$ (измерено на интерферометре ZYGO PTI-250) при $\Delta_{e t}=18 \mathrm{~mm}$ приведены в табл. 5.

После проведения серии из $N=40$ измерений (20 для положительных смещений $\Delta$ и 20 для отрицательных), сделанных для $493.3 \mathrm{~mm}$ зеркала при указанных выше параметрах эталонного зеркала и устройства перемещения, расчетная погрешность измерения $\sigma_{N}(14)$ составила $0.197 \mathrm{~mm}$. Зеркала с радиусом $174.96 \mathrm{~mm}-$ $\sigma=0.1 \mathrm{~mm}$. Относительная погрешность измерения радиуса для других зеркал приведена в табл. 6.

Таким образом, исследование показало, что при использовании датчиков волнового фронта в качестве основного регистрирующего элемента в измерительном приборе можно добиться достаточно высокой точности определения радиусов кривизны оптических деталей, т. е. погрешности измерения $\leq 0.1 \%$. Дальнейшие расчеты показывают, что при увеличении перемещения $\Delta$ до 40-50 mm погрешность измерения радиуса зеркала может составить менее $0.1 \mathrm{~mm}$ для зеркал с радиусом до $1 \mathrm{~m}$. 


\section{Конфликт интересов}

Авторы заявляют, что у них нет конфликта интересов.

\section{Список литературы}

[1] Nikitin A., Sheldakova J., Kudryashov A., Denisov D., Karasik V., Sakharov A. // Proc. SPIE. 2015. V. 9369. P. 936905-1. doi 10.1117/12.2085263.

[2] Srivastava A.K., Sati K.C., Satyander Kumar // Int. J. Scientific \& Engineering Research. 2017. V. 8. N 7. P. 1752.

[3] Yang Z., Gao Z., Yuan Q. // Opt. and Las. in Engin. 2014. V. 56. P. 35. doi 10.1016/j.optlaseng.2013.12.008

[4] Kovalev M.S., Krasin G.K., Odinokov S.B., Solomashenko A.B., Zlokazov E.Yu. // Opt. Express. 2019. V. 27. P. 1563. doi 10.1364/OE.27.001563

[5] Platt B.C., Shack R. // J. Refract. Surg. 2001. V. 17. N 5. P. 573. doi 10.3928/1081-597X-20010901-13.

[6] Southwell W. // J. Opt. Soc. Am. 1980. V. 70 N 8. P. 998. doi 10.1364/JOSA.70.000998

[7] Компания „Активная Оптика“Датчик Шака-Гартмана WFS-11.26-3.2-0.136 [Электронный ресурс] Режим доступа: http://www.activeoptics.ru/files/products/files/shws/ shws.htm\#show11.

[8] Neal D.R., Copland J., Neal D.A. // Proc. SPIE. 2002. V. 4779. P. 148. doi 10.1117/12.450850.

[9] Chernyshov A., Sterr U., Riehle F., Helmcke J., Pfund J. // Appl. Opt. 2005. V. 44. N 30. P. 6419. doi 10.1364/AO.44.006419

[10] Nikitin A., Baryshnikov N., Denisov D., et.al. // Proc. SPIE. 2018. V. 10539. P. 105390Z-1. doi 10.1117/12.2297078

[11] Барышников Н.В., Денисов Д.Г., Карасик В.Е., Сахаров A.A. // Сб. трудов IX Международной конференции молодых ученых и специалистов „Оптика - 2015“ г. Санкт-Петербург:: Университет ИТМО, 2015. С. 508.

[12] Zygo Corporation, PTI 250 Operating Manual OMP-0490G. 2004. P. 7.

[13] Thorlabs Linear Translation Stage. [Электронный pecypc] Режим доступа: https://www.thorlabs.com/ newgrouppage $9 . c f m ?$ objectgroup_id $=5697$

[14] Laser Components Translation Stage [Электронный pecypc] Режим доступа: https://asercomponents.ru/catalog/ optomecanika/motorizovannye-translyacionnye-stoliki

[15] Neal D.R., Copland J., Neal D.A., Topa D.M., Riera P. Measurement of lens focal length using multi-curvature analysis of Shack-Hartmann wavefront data [Электронный pecypc] Режим доступа: https://citeseerx.ist.psu.edu/viewdoc/ download?doi=10.1.1.513.8016\&rep=rep1\&type $=$ pdf.

[16] Барышников Н.В., Животовский И.В., Карасик В.Е., Сахаров А.А. // Контенант. 2018. Т. 17. № 3. С. 129.

[17] Radiant Zemax, Zemax User's Manual, Radiant Zemax LLC. 2012. P. 205.

[18] Барышников Н.В., Денисов Д.Г., Животовский И.В. и др. Патент РФ № 2667323, 2009.

[19] Bevington P.R., Robinson D.K. Data Reduction and Error Analysis for the Physical Sciences. McGraw-Hill, 1969. 336 p. 